\title{
PERFORMANCE OF A FADE-COMPENSATED 16QAM WITH DIVERSITY RECEPTION IN MOBILE RADIO CHANNELS
}

\author{
H. K. Lau and S. W. Cheung \\ Department of Electrical and Electronic Engineering \\ The University of Hong Kong \\ Pokfulam Road \\ HONG KONG
}

\begin{abstract}
The paper studies the bit-error-rate (BER) performance of a fade-compensated 16QAM with two-branch postdetection selection combining diversity reception in the Rayleigh fading channels. A pilot symbol-aided (PSA) technique that uses both pilot symbols and data symbols is employed for the fading compensation. Computer simulation results have shown that, the use of diversity reception technique can significantly improve the BER performance when the normalized delay between the signals at the two receivers is small. It is also shown that, the error-floor is sensitive to the normalized delay, but relatively less sensitive to the power ratio between the signals at the two receivers.
\end{abstract}

\section{INTRODUCTION}

To cope with the ever-increasing demands for system capacity, amplitude-phase modulated signals are expected to be used to increase the spectral efficiency. Among these signals, the 16-ary quadrature-amplitude modulation (16QAM) is one of the promising candidate [1]. However, due to the wide tracking bandwidth requirements, efficient transmission of this signal in mobile environments cannot be easily achieved. Recently, pilot symbol-aided (PSA) techniques have been proposed to enable coherent transmission of digital signals over the fading channels [2-8]. In a PSA system, the data symbol sequence is divided into frames of symbols for transmission. A known pilot symbol is inserted at the beginning of each frame. At the receiver, these pilot symbols are extracted and used to estimate the fading distortions in the data symbols. In previous studies [2-6], the fading estimation techniques make use of only the pilot symbols but ignore the fading information in the data symbols. Estimation techniques that use both the pilot symbols and data symbols have been proposed and results have shown that, in both the frequency non-selective and frequency selective Rayleigh fading channels, substantial improvements on the BER performances can be achieved [7-8].

It is known that, the use of diversity reception technique can significantly improve the BER performances of the systems in multipath fading environments [9-10]. However, little work has been done on the performances of PSA systems with diversity reception. This paper studies the effectiveness of incorporating a two-branch postdetection selection combining diversity reception technique in a PSA-16QAM system. Simulation results have shown that, when normalized delay between the signals at the two receivers is small, the use of diversity technique can dramatically reduce the error-floor by more than three orders of magnitude (from $5.1 \times 10^{-3}$ to $1.8 \times 10^{-6}$, when the normalized delay equals 0.125 , and the signal powers at the two receivers are equal). As the normalized delay increases, performance gain obtained from the diversity reception technique decreases. All these observations agree with previous studies on the diversity reception techniques in non-PSA systems [9-10].

\section{SYSTEM MODEL}

The baseband equivalent model of the data-transmission system used in the study is shown in Fig. 1. The information to be transmitted is carried by the binary digits $\left\{u_{n}\right\}$. When the encoder has received the binary information $\left\{u_{n}\right\}$ at time $t=n T$ seconds (where $n$ is a positive integer and $T$ is the symbol duration), it maps these signals into a data symbol $\left\{d_{k, i}\right\}$ according to the $16 \mathrm{QAM}$ signal constellations.

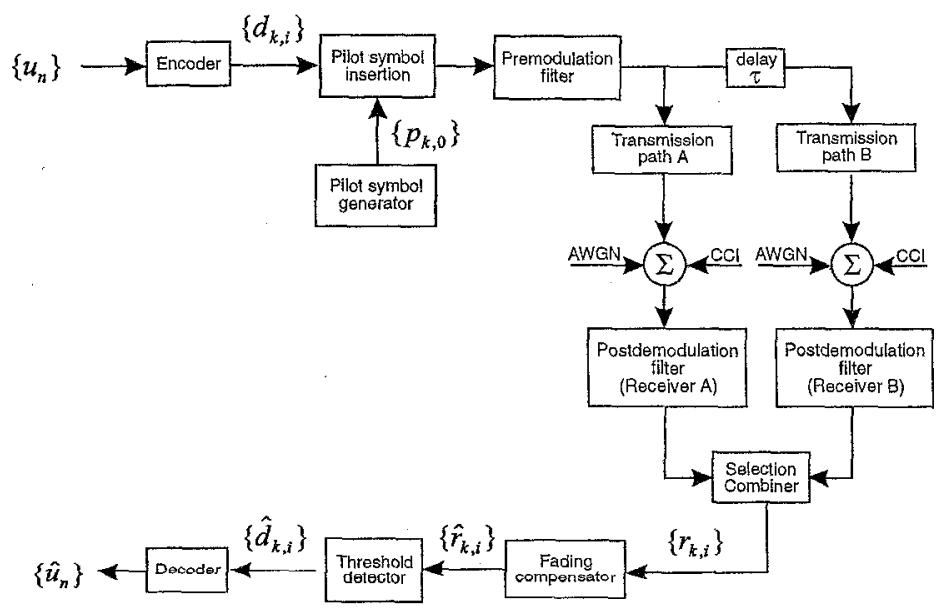

Fig. 1 Baseband Equivalent Model of the System

For every $(L-1)$-data symbols, a pilot symbol from a known pseudorandom-symbol sequence $\left\{p_{k, 0}\right\}$ is inserted at the beginning of the frame, to form an $L$-symbol frame as shown in Fig. 2. 


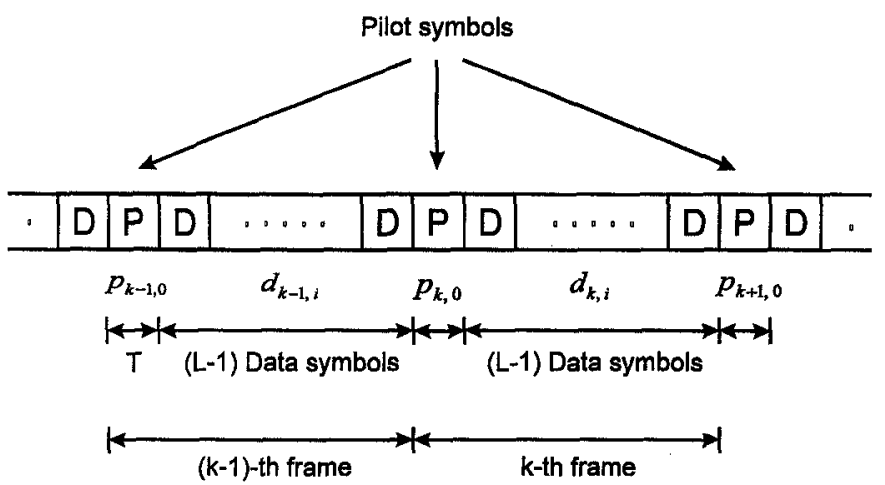

Fig. 2 Frame Structure of Transmitted Symbol Sequence

A pseudorandom sequence of pilot symbols is used to avoid transmitting tones [3]. To minimize the performance degradation due to additive white Gaussian noise (AWGN), pilot symbols are chosen from those signal vectors with the largest energy level in the signal constellation [6]. At time $t=n T$ seconds, the symbol to be transmitted is used to form the impulse $q_{n} \delta(t-n T)$, which is fed to the premodulation filter with an impulse response $a(t)$. The $q_{n}$ is complex-valued and is either a data symbol or a pilot symbol, and $\delta(t)$ is the Dirac delta function. At the output of the premodulation filter, the signal becomes $\Sigma_{n} q_{n} a(t-n T)$ and is then used to linearly modulate a carrier signal to produce the transmit signal.

The transmission paths in Fig. 1 are mobile radio channels that introduce uncorrelated Rayleigh fading distortion to the input signals. The AWGN and the co-channel-interference (CCI) are assumed to be added at the input of the receivers. The combination of the signals from the two receivers (receiver A and receiver B) accounts for the two-branch diversity reception. Since the PSA fading compensation is employed in the system (fading distortions in pilot symbols are used to estimate the fading distortion in data symbols), ordinary selection combining criterion of data symbols is not appropriate. The combiner circuit would not simply select the stronger signal from the two receivers on symbol basis. Instead, selection would be on frame basis (i.e., the whole frame of data symbols from one of the receiver is selected, not the individual data symbol). The selection would depend on the fading distortion experienced in the pilot symbols from the two receivers. Detailed fading compensation technique and selection combining criteria are discussed in section III.

\section{FADING COMPENSATION AND SELECTION COMBINING}

The signals at the $i$-th position of the $k$-th received frame at receiver $\mathrm{A}$ and receiver $\mathrm{B}$ can be written as, respectively,

$$
\begin{aligned}
r_{k, i}^{A} & =q_{k, i} y_{k, i}^{A}+w_{k, i}^{A}+c_{k, i}^{A} \\
r_{k, i}^{B} & =q_{k, i} y_{k, i}^{B}+w_{k, i}^{B}+c_{k, i}^{B}
\end{aligned}
$$

where $q_{k, i}$ is either a transmitted pilot symbol or a transmitted data symbol, $y_{k, i}$ is the change (fading distortion), $w_{k, i}$ is the AWGN, and $c_{k, i}$ is the interference (CCI), at the $i$-th symbol of the $k$-th frame. The superscripts ( $A$ or $B$ ) identify the signal from receiver $\mathrm{A}$ or receiver $\mathrm{B}$. For $i=0$, the signals at the two receivers are

$$
\begin{aligned}
& r_{k, 0}^{A}=p_{k, 0} y_{k, 0}^{A}+w_{k, 0}^{A}+c_{k, 0}^{A} \\
& r_{k, 0}^{B}=p_{k, 0} y_{k, 0}^{B}+w_{k, 0}^{B}+c_{k, 0}^{B}
\end{aligned}
$$

where $p_{k, 0}$ is the pilot symbol in the $k$-th frame. For $i=1,2, \ldots, L-1$, the signals at two receivers are

$$
\begin{aligned}
r_{k, i}^{A} & =d_{k, i} y_{k, i}^{A}+w_{k, i}^{A}+c_{k, i}^{A} \\
r_{k, i}^{B} & =d_{k, i} y_{k, i}^{B}+w_{k, i}^{B}+c^{B}{ }_{k, i}
\end{aligned}
$$

where $d_{k, i}$ is a data symbol at the $i$-th symbol of the $k$-th frame. Since the pilot symbol $p_{k, 0}$ is known at the receiver, $y_{k, 0}^{A}$ and $y_{k, 0}^{B}$ can be obtained using (1c) and (1d) as

$$
\begin{aligned}
y_{k, 0}^{A} & =\frac{r_{k, 0}^{A}}{p_{k, 0}}-\frac{w_{k, 0}^{A}}{p_{k, 0}}-\frac{c_{k, 0}^{A}}{p_{k, 0}} \\
y_{k, 0}^{B} & =\frac{r_{k, 0}^{B}}{p_{k, 0}}-\frac{w_{k, 0}^{B}}{p_{k, 0}}-\frac{c_{k, 0}^{B}}{p_{k, 0}}
\end{aligned}
$$

At low noise and low interference environments, $y_{k, 0}^{A}$, $y^{A}{ }_{k+1,0}, y_{k, 0}^{B}$, and $y^{B}{ }_{k+1,0}$ can be estimated as

$$
\begin{aligned}
\hat{y}_{k, 0}^{A} & =\frac{r_{k, 0}^{A}}{p_{k, 0}} \\
\hat{y}_{k+1,0}^{A} & =\frac{r^{A}{ }_{k+1,0}}{p_{k+1,0}} \\
\hat{y}^{B}{ }_{k, 0} & =\frac{r^{B}{ }_{k, 0}}{p_{k, 0}} \\
\hat{y}^{B}{ }_{k+1,0} & =\frac{r^{B}{ }_{k+1,0}}{p_{k+1,0}}
\end{aligned}
$$

\section{Selection Combining}

A simple two-branch postdetection selection combining diversity reception technique is used to select appropriate signal (on the frame basis) from the two receivers. Since the fading compensation process of the data symbols base on the fading distortion experienced in the pilot symbols, the proposed selection criteria would not simply base on the magnitude of the individual symbols of the received signal. Instead, it depends on the magnitude of the two pilot symbols in the $k$-th and $(k+1)$-th frame. Since the transmitted pilot symbols are selected to have the same signal energy, the selection could base on the estimated fading distortion on the two pilot symbols. The selection would base on (4a) and (4b).

$$
\begin{aligned}
& \left|\operatorname{Re}\left(\hat{y}_{k, 0}^{A}+\hat{y}_{k+1,0}^{A}\right)\right|+\left|\operatorname{Im}\left(\hat{y}_{k, 0}^{A}+\hat{y}^{A}{ }_{k+1,0}\right)\right| \\
& \left|\operatorname{Re}\left(\hat{y}_{k, 0}^{B}+\hat{y}_{k+1,0}^{B}\right)\right|+\left|\operatorname{Im}\left(\hat{y}_{k, 0}^{B}+\hat{y}^{B}{ }_{k+1,0}\right)\right|
\end{aligned}
$$

If (4a) is greater than (4b), the combiner circuit will select the signal (i.e., $r_{k, i}$, for $i=1$ to $L-1$ ) from receiver A for the $k$-th frame. If (4a) is smaller than (4b), signal for the $k$-th frame will be selected from receiver B. Finally, if (4a) and (4b) are equal, the combiner circuit will randomly select either receiver 
$\mathrm{A}$ or receiver $\mathrm{B}$. Once the appropriate data symbols for the $k$-th frame is selected, fading compensation process for the $k$-th frame begins. In the following analysis, notations without the superscript $A$ or $B$ are refer to the selected signal from the combiner circuit (either from receiver $A$ or receiver $B$ ).

The proposed compensation process is suitable for frame lengths of $4,8, \ldots$, or $2^{m}$ (where $m$ is an integer), and consists of two compensation stages. The first stage works on the data symbols in the even-number positions (i.e., $i=2,4, \ldots, L-2$ ), while the second stage works on the data symbols in the oddnumber positions (i.e., $i=1,3, \ldots, L-1$ ) of a frame.

\section{Compensation of Data Symbols in Even-Number Positions}

The changes on the pilot symbols due to fading distortion in the $k$-th frame, $\hat{y}_{k, 0}$, and in the $(k+1)$-th frame, $\hat{y}_{k+1,0}$, can be obtained using (3). The estimate of $y_{k, L / 2}$ is obtained as

$$
\tilde{y}_{k, L / 2}=\frac{\hat{y}_{k, 0}+\hat{y}_{k+1,0}}{2}
$$

This signal is then used to correct the fading effects in $r_{k, L / 2}$ to give an estimate of the data signal

$$
\hat{r}_{k, L / 2}=\frac{r_{k, L / 2}}{\tilde{y}_{k, L / 2}}
$$

which is threshold detected to produce the data symbol $\hat{d}_{k, L / 2}$ (where $\hat{d}_{k, L / 2}$ is one of the vector in the signal constellation). A more accurate fading distortion in $r_{k, L / 2}$ is then given by $r_{k, L / 2} / \hat{d}_{k, L / 2}$, rather than $\tilde{y}_{k, L / 2}$.

If $L=4$, the compensation process for the data symbols in the even-number positions is completed and the compensation process for the data symbols in the odd-number positions begins. However, if $L=8$, the compensation process for the data symbols in the even-number positions continues as follows. The estimates of $y_{k, L / 4}$ and $y_{k, 3 L / 4}$ can be obtained as, respectively,

$$
\begin{gathered}
\tilde{y}_{k, L / 4}=\frac{1}{2}\left(\hat{y}_{k, 0}+\frac{r_{k, L / 2}}{\hat{d}_{k, L / 2}}\right) \\
\tilde{y}_{k, 3 L / 4}=\frac{1}{2}\left(\frac{r_{k, L / 2}}{\hat{d}_{k, L / 2}}+\hat{y}_{k+1,0}\right)
\end{gathered}
$$

These signals $\tilde{y}_{k, L / 4}$ and $\tilde{y}_{k, 3 L / 4}$ are used to correct $r_{k, L / 4}$ and $r_{k, 3 L / 4}$, respectively, to obtain the signals

$$
\begin{aligned}
\hat{r}_{k, L / 4} & =\frac{r_{k, L / 4}}{\tilde{y}_{k, L / 4}} \\
\hat{r}_{k, 3 L / 4} & =\frac{r_{k, 3 L / 4}}{\tilde{y}_{k, 3 L / 4}}
\end{aligned}
$$

which are used to obtain the detected data symbols $\hat{d}_{k, L / 4}$ and $\hat{d}_{k, 3 L / 4}$.

If $L=16$, the compensation process continues further. The corrected signals, $\hat{d}_{k, L / 4}$ and $\hat{d}_{k, 3 L / 4}$, are used together with the pilot symbols, $p_{k, 0}$ and $p_{k+1,0}$, to correct the data symbols according to

$$
\begin{gathered}
\tilde{y}_{k, L / 8}=\frac{1}{2}\left(\hat{y}_{k, 0}+\frac{r_{k, L / 4}}{\hat{d}_{k, L / 4}}\right) \\
\tilde{y}_{k, 7 L / 8}=\frac{1}{2}\left(\frac{r_{k, 3 L / 4}}{\hat{d}_{k, 3 L / 4}}+\hat{y}_{k+1,0}\right) \\
\tilde{y}_{k, j L / 8}=\frac{1}{2}\left(\frac{r_{k,(j-1) L / 8}}{\hat{d}_{k,(j-1) L / 8}}+\frac{r_{k,(j+1) L / 8}}{\hat{d}_{k,(j+1) L / 8}}\right) \text { for } j=3,5
\end{gathered}
$$

Similar equations can be derived for $L=32,64, \ldots, 2^{m}$. The compensation process continues until all the data symbols in the even-number positions are done. Then the compensation process for the data symbols in the odd-number positions begins.

\section{Compensation of Data Symbols in Odd-Number Positions}

For compensation of the data symbols in odd-number positions, $\tilde{y}_{k, i}$ and $\hat{r}_{k, i}$ are obtained as

$$
\begin{gathered}
\tilde{y}_{k, 1}=\frac{1}{2}\left(\hat{y}_{k, 0}+\frac{r_{k, 2}}{\hat{d}_{k, 2}}\right) \\
\tilde{y}_{k, L-1}=\frac{1}{2}\left(\frac{r_{k, L-2}}{\hat{d}_{k, L-2}}+\hat{y}_{k+1,0}\right) \\
\tilde{y}_{k, j}=\frac{1}{2}\left(\frac{r_{k, j-1}}{\hat{d}_{k, j-1}}+\frac{r_{k, j+1}}{\hat{d}_{k, j+1}}\right) \text { for } j=3,5, \ldots, L-3 \\
\hat{r}_{k, i}=\frac{r_{k, i}}{\tilde{y}_{k, i}} \quad \text { for } i=1,3, \ldots, L-1
\end{gathered}
$$

All the corrected signal samples $\left\{\hat{r}_{k, i}\right\}$ are fed to the threshold detector to produce $\left\{\hat{d}_{k, i}\right\}$ which are finally decoded into the binary data $\left\{\hat{u}_{n}\right\}$. The whole compensation process, including the selection combining process, repeats for all the received frames.

\section{Energy Loss Due to Transmission of Pilot Symbols}

Since the pilot symbols are known to the receiver, they carry no data information. However, the pilot symbols require a certain amount of power for transmission. Thus for a system with a fixed transmission power, a portion of the power has to be assigned for transmitting the pilot symbols. Therefore, the net average data-symbol energy is reduced. If the same transmit data-symbol energy as that without transmitting the pilot symbols is to be maintained, the average energy per data symbol has to be increased by [7]

$$
\Delta E_{s}=10 \log \left[\left(\frac{\left|P_{p}\right|}{\left|P_{d}\right|}\right)\left(\frac{1}{L-1}\right)+1\right]
$$

where $L$ is the frame length, $\left|P_{p}\right|$ and $\left|P_{d}\right|$ are the average energies required to transmit a pilot symbol and a data symbol, 
respectively. Since the pilot symbols are selected from those signal vectors with the largest signal levels in the constellation, $\left|P_{p}\right|:\left|P_{d}\right|=9: 5$ and $(9 \mathrm{a})$ becomes

$$
\Delta E_{\mathrm{s}}=10 \log \left[\frac{5 L+4}{5(L-1)}\right]
$$

Taking into account of the loss of energy due to transmitting the pilot symbols, the BER performance of the coherent transmission of 16QAM over a Gaussian channel, with different values of frame length $L$, become [7]

$$
P_{b, 16 \mathrm{QAM}} \approx \frac{3}{8} \operatorname{erfc}\left[\sqrt{\frac{6}{15}\left(\frac{E_{b}}{N_{0}}-\Delta E_{s}\right)}\right] \times\left\{1-\frac{3}{8} \operatorname{erfc}\left[\sqrt{\frac{6}{15}\left(\frac{E_{b}}{N_{0}}-\Delta E_{s}\right)}\right]\right\}
$$

The relationship between $P_{b, 16 Q A M}$ and $E_{b} / N_{0}$ in (10) is shown in Fig. 3. No compensation or diversity reception technique is used in the system. It can be seen that, the longer the frame length $L$ is, the less is the performance degradation due to transmitting the pilot symbols.

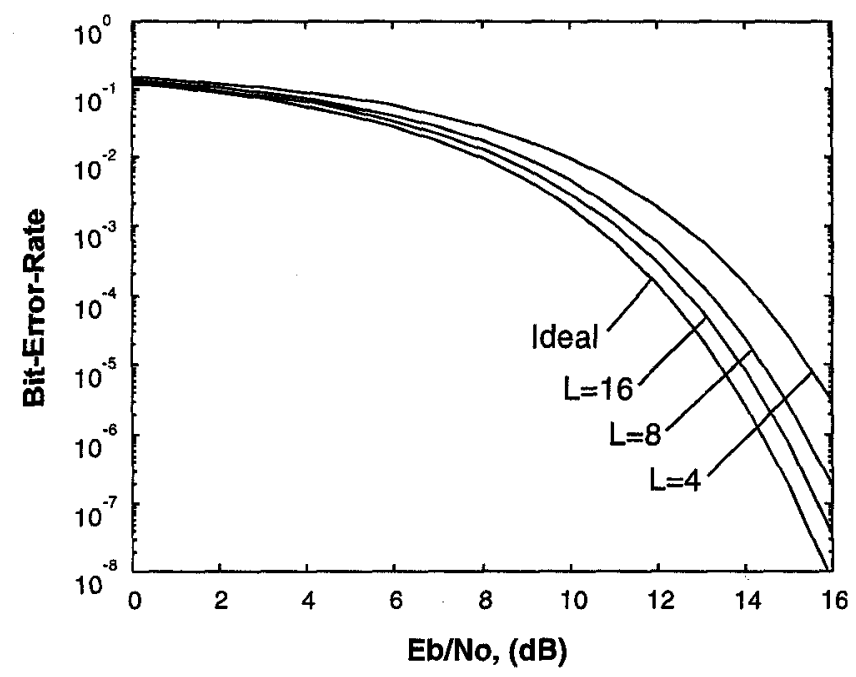

Fig. 3 Performance of 16QAM in a Gaussian Channel

\section{Bandwidth Considerations}

In addition to signal energy, bandwidth is also required to transmit the pilot symbols. If the same system throughput as that without transmitting the pilot symbols is to be maintained, the resultant symbol rate needs to be increased by a factor of $L /(L-1)$. Obviously, signal with a longer frame length requires less amount of extra bandwidth and extra energy for transmitting the pilot symbols.

\section{RESULTS AND DISCUSSIONS}

The signal-to-noise ratio is defined as

$$
\mathrm{SNR}=10 \log \left(\frac{E_{b}}{N_{0}}\right)
$$

where $E_{b}$ is the average transmitted bit energy and $N_{0}$ is the single-sided power spectral density of the AWGN. The power ratio of the signals at two receivers is defined as

$$
\mathrm{SDR}=10 \log \left(\frac{S}{D}\right)
$$

where $S$ and $D$ are the received signal power at receiver A and $B$, respectively. The signal-to-interference power ratio is defined as

$$
\mathrm{SIR}=10 \log \left(\frac{S}{I}\right)
$$

where $I$ is the power of the interference $(\mathrm{CCI})$ signal. The normalized delay is defined as

$$
\tau / T
$$

where $\tau$ is the time-delay of the signal from receiver B with reference to the signal at receiver $\mathrm{A}$, and $T$ is the symbol duration.

A series of computer-simulation tests has been carried out to investigate the effectiveness of incorporating the two-branch postdetection selection combining diversity reception with PSA technique in the mobile radio channels. Throughout the tests, a normalized Doppler spread of 0.005 (correspond to a transmission rate of $32 \mathrm{kbit} / \mathrm{s}$ at a traveling velocity of $48 \mathrm{~km} / \mathrm{hr}$ and carrier frequency of $900 \mathrm{MHz}$ ) and a frame length of $L=16$ are used. When $L=16$, only a $0.49 \mathrm{~dB}$ energy loss and a $6.25 \%$ throughput degradation are caused by transmitting the pilot symbols.

The results on the BER performances of the 16QAM signals against SNR and SIR are shown in Figs. 4 and 5, respectively. The error-floors, i.e., $S N R=\infty \mathrm{dB}$, against the SDR, and against the $\tau / T$ (normalized delay), are shown in Figs. 6 and 7, respectively. It is shown in Figs. 4 and 5 that, the diversity technique can significantly improve the BER performance of the signal by more than two orders of magnitude at low noise (from $1.5 \times 10^{-4}$ to $1.3 \times 10^{-6}$ at $\mathrm{SNR}=40 \mathrm{~dB}$, as in Fig. 4) or low interference (from $5 \times 10^{-4}$ to $5 \times 10^{-6}$ at $\operatorname{SIR}=40 \mathrm{~dB}$, as in Fig. 5) environments.

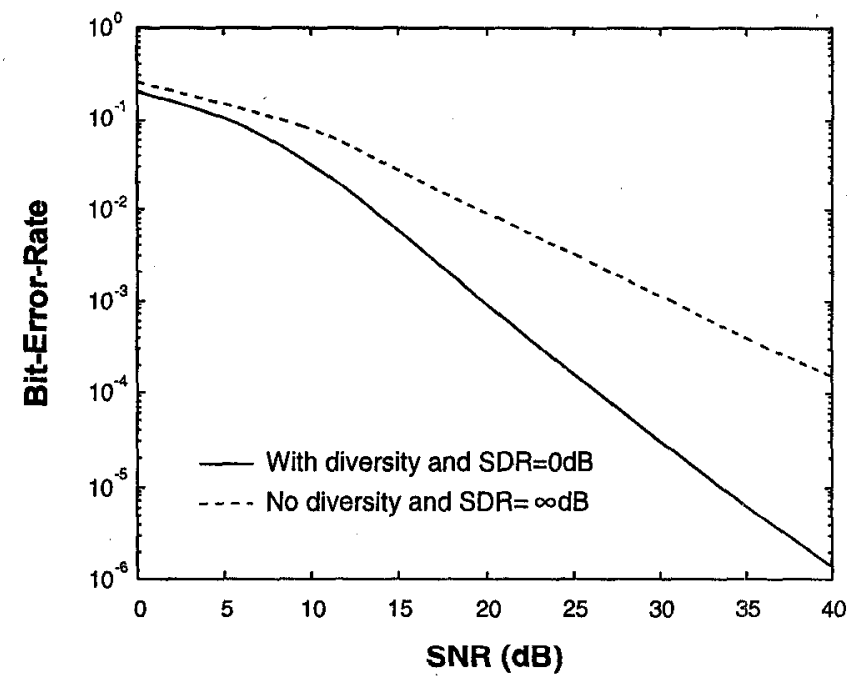

Fig. 4 BER Performances against SNR with $\mathrm{SIR}=\infty \mathrm{dB}$ 


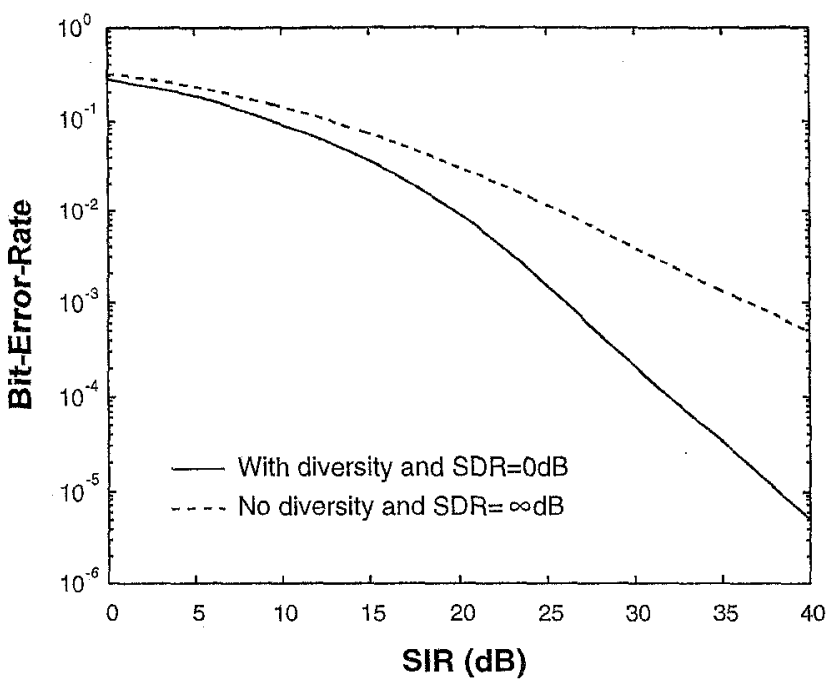

Fig. 5 BER Performances against $\mathrm{SIR}$ with $\mathrm{SNR}=\infty \mathrm{dB}$

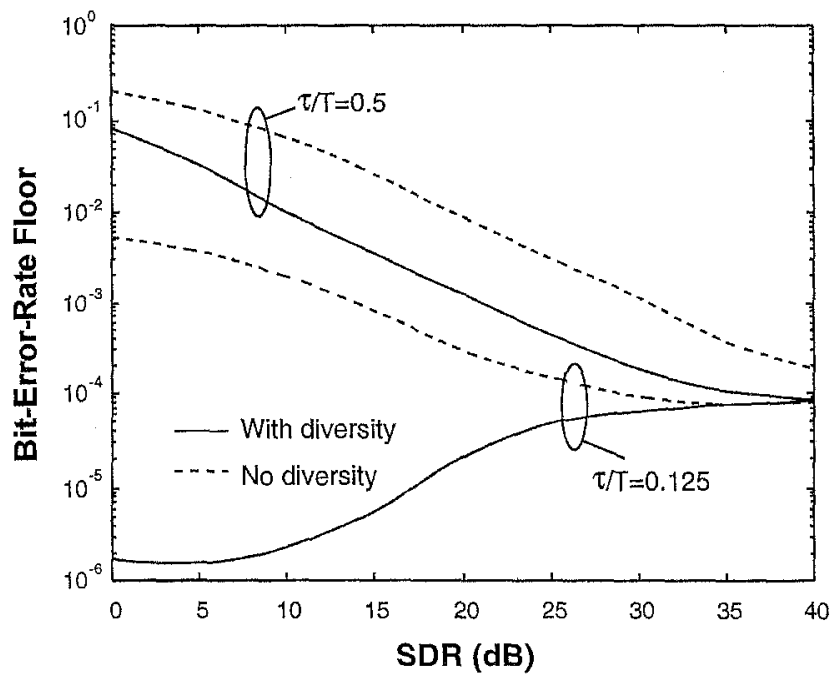

Fig. 6 BER Floors against $\mathrm{SDR}$ with $\mathrm{SNR}=\infty \mathrm{dB}$ and $\mathrm{SIR}=\infty \mathrm{dB}$

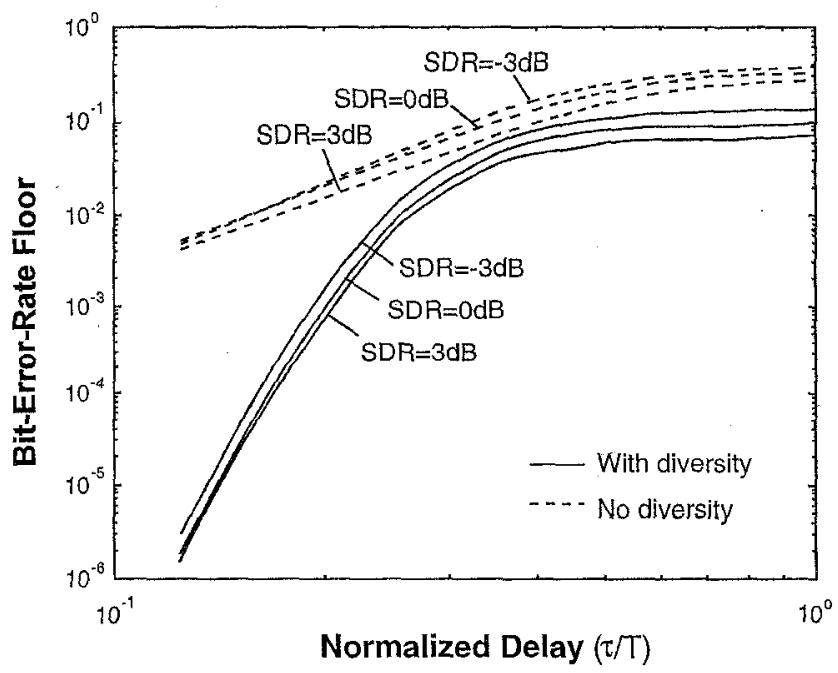

Fig. 7 BER Floors against $\tau / T$ with $\mathrm{SNR}=\infty \mathrm{dB}$ and $\mathrm{SIR}=\infty \mathrm{dB}$
When the normalized delay between the signals from the two receivers is small, the use of diversity technique can dramatically reduce the error-floor by more than three orders of magnitude (from $5.1 \times 10^{-3}$ to $1.8 \times 10^{-6}$ at $\tau / T=0.125$ and $\mathrm{SDR}=0 \mathrm{~dB}$, as in Fig. 6).

However, it is also shown in Figs. 6 and 7 that, the diversity improvement diminishes as the normalized delay increases. In addition, the error-floor is sensitive to the normalized delay, but relatively less sensitive to the power ratio between the signals at the two receivers. These observations agree with the diversity reception technique used in non-PSA systems.

\section{CONCLUSIONS}

The effectiveness of incorporating a two-branch postdetection selection combining diversity reception technique in a PSA-16QAM system is investigated. The PSA technique uses both the pilot symbols and data symbols for fading compensation. When a frame length of $L=16$ is used, only small energy loss $(0.49 \mathrm{~dB})$ and throughput degradation $(6.25 \%)$ are caused by transmitting the pilot symbols. Computer simulation results have shown that, the use of diversity technique can dramatically improve the BER performance by more than three orders of magnitude when the normalized delay is small. As the delay increases, the improvement gain obtained from diversity reception decreases. It is also shown that, the error floor is sensitive to the normalized delay, but relatively less sensitive to the power ratio of the signals at two receivers.

\section{REFERENCES}

[1] W. T. Webb and L. Hanzo, Modern Quadrature Amplitude Modulation, Pentech Press, 1994.

[2] M. L. Moher and J. H. Lodge, "TCMP-A Modulation and Coding Strategy for Rician Fading Channel," IEEE J. on Sel. Areas in Commun., vol. 7, no. 9, pp. 1347-1355, Dec. 1989.

[3] J. K. Cavers, "An Analysis of Pilot Symbol Assisted Modulation for Rayleigh Fading Channels," IEEE Trans. Veh. Technol., vol. 40, no. 4, pp. 686-693, Nov. 1991.

[4] S. Sampei and T. Sunaga, "Rayleigh Fading Compensation for QAM in Land Mobile Radio Communications," IEEE Trans. Veh. Technol, vol. 42, no. 2, pp. 137-147, May 1993.

[5] C. L. Liu and K. Feher, "Pilot-Symbol Aided Coherent $M$-ary PSK in Frequency-Selective Fast Rayleigh Fading Channels," IEEE Trans. Commun., vol. 42, no. 1, pp. 54-62, Jan. 1994.

[6] H. K. Lau and S. W. Cheung, "A Pilot Symböl-Aided Technique Used for Digital Signals in Multipath Environments," in Proc. of IEEE Int'l Conf. on Commun., New Orleans, USA, May 1994, pp. 1126-1130.

[7] H. K. Latr and S. W. Cheung, "A Fade-Compensation Technique for Digital Land Mobile Satellite Systems," accepted, to appear in Int $l$. J. of Sat. Commun.

[8] H. K. Lau and S. W. Cheung, "Performance of a Pilot Symbol-Aided Technique in Frequency-Selective Rayleigh Fading Channels Corrupted by Co-Channel Interference and Gaussian Noise," in Proc. of IEEE Veh. Technol. Conf., Atlanta, USA, April 1996, pp. 1008-1012.

[9] W. C. Jakes, Microwave Mobile Communications, New York, John Wiley \& Sons, 1974.

[10] F. Adachi and K. Ohno, "BER Performance of QDPSK with Postdetection Selection Diversity Reception in Mobile Radio Channels," IEEE Trans. Veh. Technol, vol. 40, no. 1, pp. 237-249, Feb. 1991. 